\title{
Aproximación a la articulación conceptual entre sustentabilidad, desarrollo humano y género
}

\author{
Approach to the conceptual articulation between sustainability, \\ human development and gender
}

Alberto Niño Cruz ${ }^{1}$, Ignacio Luna Espinoza ${ }^{2}$ y Noé Hernández Cortez

Fecha de recepción: 3 de octubre de 2020

Fecha de aceptación: 27 de enero de 2021

1- Nacionalidad: Mexicana. Grado: Maestría en Desarrollo Regional Sustentable. Adscripción: El Colegio de Veracruz. (iD) ORCID: https://orcid.org/0000-0001-6440-5101. Correo electrónico: alberto.nic@gmail.com

2- Nacionalidad: Mexicana. Grado: Maestro en Estadística. Adscripción: Universidad del Istmo. (DORCID: https://orcid.org/00000002-0686-696X. Correo electrónico: iluna@bianni.unismto.edu.mx

3- Nacionalidad: Mexicana. Grado: Doctor de Investigación en Ciencia Política. Adscripción: Universidad Autónoma de Zacatecas. DORCID: https://orcid.org/0000-0002-3079-1152. Correo electrónico: noe.hernandez@uaz.edu.mx 


\section{Resumen}

Este artículo analiza la relación teórico-conceptual entre el desarrollo humano relativo al género y la violencia de género feminicida, como componentes para medir la dimensión social del desarrollo sustentable. Para ello, se revisan las matrices teóricas en que se sustentan tales conceptos, mapeando las conexiones lógicas para tener una visión más integral del desarrollo sustentable con perspectiva de género. La conclusión a la que se llega es que la violencia de género, al no incluirse como factor de impacto negativo en el Índice de Desarrollo Humano Relativo al Género, no retrata de forma realista la dimensión social del desarrollo sustentable con perspectiva de género.

Palabras clave: desarrollo sustentable, desarrollo humano relativo al género, violencia de género, feminicidio.

\section{Abstract}

This article analyzes the theoretical-conceptual relationship between human development related to gender and feminicidal gender violence as the components to measure the social dimension of sustainable development. To do so, the theoretical matrices on which such concepts are based were reviewed, mapping the logical connections to have a more comprehensive vision of sustainable development with a gender perspective. The reached conclusion is that, since gender violence is not included as a negative impact factor in the Human Development Index Relative to Gender, it does not realistically portrays the social dimension of sustainable development with a gender perspective.

Keywords: sustainable development, human development related to gender, gender violence, femicide. 


\section{Introducción}

E

1 feminicidio constituye un fenómeno global que se ha incrementado de manera alarmante en los últimos años. La lucha de familiares de víctimas, investigadoras y colectivos de mujeres en todo el mundo ha contribuido a su visibilidad, a pesar de resistencias colocadas en multitud de trincheras -incluidas instancias gubernamentales-. De igual manera, desde innumerables posiciones ciudadanas, institucionales, académicas y políticas se han venido acumulando miradas centradas en la denuncia, el análisis y la comprensión de la violencia de género y su expresión extrema, con el propósito de identificar los mecanismos socioculturales, jurídicos y políticos que la cultivan o pretenden ocultarla y que generan impunidad. Este trabajo pretende sumarse a tales esfuerzos.

La investigación se posiciona en la intersección de tres constructos teóricos que, si bien tienen antecedentes lejanos, encontraron legitimidad en medios intelectuales e institucionales casi simultáneamente entre los años 80 y 90 del siglo pasado, ellos son, las teorías sobre el desarrollo sustentable, el desarrollo humano y la teoría de género. El artículo gira en torno a la articulación de conceptos relevantes de las tres teorías enunciadas, buscando establecer la relación conceptual entre el Índice de Desarrollo Humano Relativo al Género, y el factor de impacto negativo de los feminicidios, que retrate de forma realista la dimensión social del desarrollo sustentable con perspectiva de género en México, lo anterior con base en la crítica formulada en la teoría de género.

Este artículo constituye el marco teórico de una investigación en desarrollo cuyo objetivo es analizar la asociación entre el Índice de Desarrollo Humano Relativo al Género, y el factor de impacto negativo de la violencia extrema de género, el feminicidio, en las entidades federativas de la República Mexicana. En ese sentido, el artículo presenta un análisis de contenido de los enfoques teóricos pertinentes para la recuperación de los conceptos básicos y necesarios para el objetivo enunciado. Las preguntas de investigación a que responde nuestra investigación son las siguientes: ¿Qué relación existe entre el desarrollo sustentable, el desarrollo humano y la violencia de género?, y ¿qué relación puede encontrarse implicada entre el Índice de Desarrollo Humano Relativo al Género y el feminicidio?

El artículo se estructura en cuatro partes. La primera hace una revisión conceptual del desarrollo poco después de la segunda posguerra, hasta la emergencia del desarrollo sustentable como nuevo paradigma. Una segunda parte va del planteamiento de la teoría de desarrollo humano, a lo que puede considerarse su correlato como instrumento de medición del desarrollo social: el Índice de Desarrollo Humano. En la tercera parte se aborda el enfoque o perspectiva de género como herramienta analítica para la explicación de la desigualdad, la opresión y particularmente la violencia contra la mujer. Finalmente, en un cuarto apartado se presentan las conclusiones a las que se han llegado.

\section{Del desarrollo al desarrollo sustentable}

Un largo trecho se ha recorrido en las Ciencias Sociales antes de arribar a la concepción de la sustentabilidad, hoy ampliamente aceptada como paradigma del desarrollo. En su origen, la cuestión del desarrollo como categoría analítica y normativa nace junto a la de subdesarrollo. De acuerdo con Vergara y Ortiz 
(2016) y Fougeyrollas (1980), esta concepción teórica hace su aparición en las postrimerías de la segunda posguerra para designar distintos grupos de países: los desarrollados y los subdesarrollados, identificados los primeros con las naciones autodenominadas occidentales, y los segundos con el resto de países.

A partir de una interpretación sociológica, Fougeyrollas (1980) sostiene que el sustrato de la emergencia de esta nueva concepción de las naciones se relaciona directamente con "el desplome del régimen capitalista en Europa del Este y China" (p. 263), lo que provocará que, ante la posibilidad de nuevas revueltas sociales en los países atrasados, se implementarán por parte de los países industrializados medidas que lo impidan. Rostow (1965), economista norteamericano impulsor de la teoría de la modernización, es reconocido como uno de los principales teóricos, o acaso el más sobresaliente, que proporciona sustento a la teoría del crecimiento en esos años, diseña una propuesta concebida en etapas secuenciales que, se argumenta, tiene la finalidad de llevar a las sociedades desde un estado inicial de completo atraso -etapa inicial-, a la "americanización" de las mismas -etapa final-, entendiendo por esto la llegada de estilos de vida similares a los de los países desarrollados. De acuerdo con Fougeyrollas (1980), la propuesta teórica de Rostow consistió en formular medidas "preventivas", con base en un modelo de crecimiento económico en cinco diferentes etapas que las naciones deben de transitar para acceder al desarrollo: "1) sociedad tradicional, 2) condiciones previas para el impulso inicial, 3) el impulso inicial, 4) la marcha hacia la madurez y 5) la era del gran consumo de masas" (p. 268). A pesar del impulso del desarrollo en los países subdesarrollados, mediante planes, programas y financiamiento, las brechas de la desigualdad se mantuvieron o ampliaron entre ambos grupos de países, dando lugar a lo que distintos autores identificados con la llamada teoría de la dependencia denominaron en los años 60 desarrollo del subdesarrollo (Frank, 1967).

Desde un enfoque más próximo a la economía, Vergara y Ortiz (2016) plantearán que existen dos grandes visiones del tema con matices en la interpretación. Quienes lo argumentan como un problema de atraso económico, y quienes postulan que es un problema estructural del desarrollo del capitalismo internacional, vale decir, que el desarrollo de unos países condena al subdesarrollo de otros, lo que dará origen a una pluralidad de herramientas analíticas.

\subsection{Desarrollo sustentable}

En el marco diverso de las teorías del desarrollo, de acuerdo con Vergara y Ortiz (2016), el desarrollo sustentable (DS) es un concepto que tomó fuerza en los años 80 con la entrada en escena de la Estrategia Mundial de Conservación, "que lo presentaba como un objetivo para ser logrado mediante la conservación de los recursos naturales" (p. 35). Asimismo, el desarrollo sustentable se considera como un modelo que "tiene sus raíces epistemológicas en el diálogo transdisciplinario con el propósito de percibir las dificultades de una forma más integral y multidimensional” (Hernández et al., 2018, p. 12).

En 1987 es presentado el documento Nuestro Futuro Común que enuncia la definición mayormente difundida de desarrollo sustentable, -por cierto, no exenta de controversia- que con el transcurso de los años se consolidó como propuesta normativa, instrumental y metodológica para el análisis del desarrollo. Emanado de este documento, el desarrollo sustentable se conceptualiza como "el desarrollo que satisface las necesidades de la generación presente sin comprometer la capacidad de las generaciones futuras para satisfacer sus propias necesidades" (ONU, 1987, p. 59). 
La generalidad de tal definición y la gran catástrofe medioambiental a la que se ha llegado, dio paso a una pluralidad de enfoques para concebir la sustentabilidad. Así, corrientes como la denominada ecología profunda que propone un biocentrismo radical, o el ecodesarrollo que postula la importancia igual entre sociedad y naturaleza, son algunas de las perspectivas desde las cuales se pretende enfrentar la crisis ambiental generada por la sociedad industrial, alentada en los últimos tiempos por su fase neoliberal.

Mención aparte merece la concepción de Foladori y Tommasino (2000) y la planteada por el ecosocialismo (Kovel y Löwy, 2001). Sostienen estas corrientes que la gran mayoría de los enfoques sobre la sustentabilidad yerra en el punto de partida al considerar la problemática ambiental sólo como un problema de relaciones técnicas, y no contemplar como trasfondo de la insustentabilidad las relaciones sociales de producción, en este caso, capitalistas, concluyendo tales posiciones en la inviabilidad de la humanidad en el marco de este sistema de relaciones.

Por encima de estos matices, o propiamente divergencias, se puede decir que ha prevalecido el acuerdo de que los componentes centrales que definen al desarrollo sustentable serían, básica o centralmente, las dimensiones económica, ambiental y social. Para decirlo esquemáticamente, se le concibe como crecimiento económico con protección ambiental y bienestar social.

\section{Otra mirada al desarrollo: de las necesidades a las capacidades y libertades}

A inicios de la década de los 90 del siglo pasado emergió la propuesta del Índice de Desarrollo Humano (IDH), de la mano con la concepción del desarrollo sustentable, como herramienta para evaluar logros, en términos de bienestar social, de personas y sociedades. En adelante será una de las principales medidas con ese propósito, desplazando la preeminencia que hasta ese entonces ocupó el producto interno bruto per cápita (PIB). La columna vertebral del IDH se encuentra en la teoría de las capacidades y libertades de Sen (2010, 2009 y 2000a,) y Nussbaum y Sen (2004), por consiguiente, destacaremos a continuación algunas de sus premisas fundamentales.

Frente a los enfoques tradicionales para conceptualizar y medir el desarrollo, consistentes en su identificación como crecimiento económico, ingreso per cápita, industrialización, modernización etc., Sen (2000b) ofrece una perspectiva diferente basada en las libertades, conceptualizando el desarrollo como "un proceso de expansión de las libertades reales que disfruta la gente" (p. 15). No es que no importe el crecimiento económico, el ingreso o la industrialización, lo que argumenta Sen (2000b) en su propuesta es que eso no basta para el desarrollo humano concebido como la expansión de las libertades humanas. Existen por supuesto otros factores imprescindibles para su logro como "satisfacer el hambre, conseguir una nutrición suficiente, tratar y prevenir enfermedades curables, tener una vivienda aceptable, disponer de medidas sanitarias adecuadas, tener acceso a agua limpia, libertad para acceder a servicios de atención social, una educación de calidad, asimismo a libertades políticas básicas" (Serret, 2008, p. 112).

$\mathrm{Al}$ enfocar el desarrollo en estos términos, lo que se propone es colocar en el centro del mismo a las personas, es decir, se busca alcanzar el bienestar social de ellas. De esta manera, las metas a las que se aspira, no es solo el crecimiento económico, sino el desarrollo de las capacidades y libertades de los individuos. 
Ahora bien, cabe preguntarse ¿cuáles son las libertades que se ven coartadas por la situación de pobreza o exclusión de acceso a servicios públicos básicos? La respuesta de Sen (2000b) es contundente: "Unas veces la falta de libertades reales se relaciona directamente con la pobreza económica, que priva a la gente de la libertad de satisfacer el hambre, alcanzar una nutrición adecuada, obtener remedios para enfermedades curables, contar con techo y abrigo, agua limpia e instalaciones sanitarias" (p. 15). Resulta prácticamente imposible, entonces, que personas con tales privaciones puedan tener la libertad real de hacer efectivos sus derechos.

Se tiene, entonces, con la teoría del desarrollo humano, una propuesta que va más allá de sólo los indicadores económicos para estimar el desarrollo de una sociedad, poniendo en el centro de la misma "la calidad de vida de las personas en todos los ámbitos de sus derechos y libertades económicas, sociales y políticas" (Serret, 2008, p. 112). Así, el desarrollo humano se concibe dentro del horizonte normativo de la justicia y la democracia, la realización plena de la vida de las personas, identificando las injusticias sobre las personas que padecen de la dominación y de las asimetrías de poder:

En verdad, las respuestas que un enfoque trascendental da, o puede dar, son muy distintas y distantes del tipo de preocupaciones que interesan a la gente en sus discusiones sobre la justicia y la injusticia del mundo (por ejemplo, las iniquidades del hambre, la pobreza, el analfabetismo, la tortura, el racismo, el sometimiento de las mujeres, el encarcelamiento arbitrario o la exclusión médica como problemas sociales que necesitan redención). (Sen, 2010, p. 126)

Desde esta perspectiva teórica-normativa el desarrollo humano se armoniza con las capacidades de las personas y el marco de derecho institucional que protege a las personas. Así, la idea de justicia se traduce en el diseño de instituciones y políticas públicas que impulsen y garanticen el desarrollo de las capacidades y por consiguiente se eleve la calidad de vida. En este contexto, Sen (1999, 2000a) ha sostenido fuertes argumentos para la protección de las capacidades y los derechos de las niñas y mujeres para el pleno desarrollo de su agencia y por consiguiente de sus libertades.

\subsection{Medir el Desarrollo Humano}

Con base en la perspectiva teórica de las capacidades se elaboró el Índice de Desarrollo Humano (IDH) como una medida más adecuada para estimar el desarrollo de las personas. Con la publicación en 1990 del primer informe sobre desarrollo humano por el Programa de las Naciones Unidas para el Desarrollo (PNUD), se inaugura este enfoque sistémico para medir el bienestar de las personas, no como el resultado de mayores ingresos o consumo de bienes y servicios, sino como un proceso que conduce a las personas a la ampliación de lo que pueden ser y hacer de acuerdo con sus intereses, en un marco de igualdad de oportunidades (PNUD, 2009). Si en el centro del desarrollo humano se encuentran las personas, de lo que se trata es hacer crecer y potenciar sus capacidades en un entorno social que les permita ponerlas en práctica: "por ejemplo, educar a una niña le proporcionará habilidades, pero de poco le servirán si no tiene acceso al empleo en el futuro, o si dichas habilidades no son las requeridas en el mercado laboral local" (PNUD, 2015, párr. 5). 
El IDH contempla para su construcción tres componentes considerados básicos o esenciales: "la posibilidad de alcanzar una vida larga y saludable, poder adquirir conocimientos individual y socialmente valiosos, y tener la oportunidad de obtener los recursos necesarios para disfrutar de un nivel de vida decoroso" (PNUD, 2003, p. 3). Lo anterior, se traduce en términos de indicadores como esperanza de vida, educación (alfabetismo y matriculación), e ingresos.

En perspectiva histórica, el Informe de 1995 incorporó por primera vez las desigualdades de género como parte sustancial en la evaluación del desarrollo humano, ofreciendo datos que reforzaron la necesidad de orientar con una perspectiva de género las estimaciones del índice en aquel momento:

Las mujeres siguen constituyendo el 70\% de los pobres del mundo y de ellas, las dos terceras partes son analfabetas. Las mujeres sólo ocupan el 14\% de los puestos administrativos y ejecutivos, el $10 \%$ de los escaños parlamentarios y el 6\% de los cargos en gabinetes nacionales. Según muchos sistemas jurídicos, aún no están en condiciones de igualdad. A menudo trabajan más horas que los hombres, pero gran parte de su trabajo sigue sin valorar, sin reconocer y sin apreciar. Y la amenaza de violencia las acosa durante toda su vida, desde la cuna hasta la tumba. (PNUD, 1995, p. iii)

A decir de De la Cruz (2007) es el primer informe que incorpora de manera específica la potenciación de las mujeres como una de sus metas. Y continúa:

El Informe plantea dos índices para medir la situación de las mujeres. El índice relacionado con la mujer (IDM) que se compone de las mismas variables que el IDH centrándose en la desigualdad entre mujeres y varones juntamente con el grado de adelanto medio de ambos sexos, y el Índice de Potenciación de la Mujer (IPM) que se compone de tres variables que reflejan la participación femenina en la adopción de decisiones políticas, su acceso a oportunidades profesionales y su capacidad de obtener ingresos. (p. 23)

Las libertades son consustanciales a la democracia moderna. En términos normativos la democracia es el conjunto de instituciones justas para que participen en igualdad de condiciones las mujeres con respecto a los hombres. De ahí que a partir del IDH se evalúe el acceso de las mujeres al desarrollo en una sociedad específica. Los esfuerzos por medir las libertades con que cuentan las mujeres en una comunidad política determinada, ha sido posible gracias a las mediciones de la agencia humana, en donde se puede observar el bienestar de las mujeres en términos de la realización de sus valores y el bienestar subjetivo del cual gozan (Alkire, 2005, 2008).

El bienestar de las mujeres potencia su capacidad de deliberar públicamente y de asumir responsabilidades políticas, en otras palabras, se logra el empoderamiento dentro de las instituciones democráticas de un país. De ahí la relevancia del índice de empoderamiento de la mujer, para observar las condiciones de participación pública de las mujeres con respecto a los hombres (Folbre, 2006; Ruppanner, 2009). Lo importante es que estos estudios han abonado, teórica y metodológicamente, a la construcción del Índice de Desarrollo Humano Relativo al Género, lo que permite tener un punto de partida bien establecido, para enriquecer este constructo teórico, con el enfoque del desarrollo sustentable con perspectiva de género y las valiosas aportaciones desde la teoría de género. 


\subsection{Libertades constitutivas e instrumentales}

Como punto de partida se recupera la idea de agencia en el discurso del desarrollo humano, entendida como la potencialidad de elegir horizontes de vida que resultan significativos para las personas; agencia, como capacidad de ser y hacer de las personas, asumiéndose como agentes activos en la construcción de sus vidas. Tal agencia encuéntrese acotado, por decirlo de alguna manera, entre las libertades reales de que se goza, y el acceso a oportunidades que permiten alcanzar aquellos objetivos de vida. Es esta agencia lo que permite identificar las libertades constitutivas e instrumentales para el desarrollo de las capacidades, centrándonos en la importancia en el ejercicio de las libertades de las niñas y mujeres (Sen, 1999, 2000a).

Ahora bien, si el IDH evalúa los tres componentes señalados (salud, educación e ingreso), vale la pena preguntarse acerca del papel que juegan las condiciones sociales generales para la consecución de las libertades que posibilitan alcanzarlos. Como resulta evidente, no será lo mismo lograr determinado nivel educativo en una sociedad desgarrada por graves conflictos internos, con problemas económico financieros severos, o con políticas públicas que limitan el acceso a los centros educativos, que en aquella con arreglos institucionales democráticos relativamente estables, o con los recursos suficientes y necesarios que le permiten atender y fomentar los procesos educativos con base en políticas públicas incluyentes.

En efecto, distintos autores advierten que en la teoría del desarrollo humano pueden distinguirse al menos dos dimensiones de las libertades. Así, Picazzo et al. (2011) retoma a Amartya Sen en su clasificación de la libertad en libertades constitutivas e instrumentales. Las primeras referidas a las libertades básicas individuales como finalidad del desarrollo humano, enfocadas a la vida misma, y las instrumentales, que son aquellas oportunidades y derechos: "que contribuyen directa o indirectamente a la libertad general de las personas y se ofrecen, a través de los sistemas económicos, sociales y políticos de los cuales dependen principalmente las libertades constitutivas básicas para su realización” (p. 258).

En la misma línea de argumentación, López-Calva y Vélez (2003) apuntan que:

Es importante hacer notar que cuando se alude a la ampliación del conjunto de opciones reales, se hace referencia no solamente a las opciones disponibles sino también a las condiciones económicas, institucionales, cognitivas y sociales con las que cuenta el individuo para ejercer su elección. (p. 7)

En esta vertiente de argumentación se puede concluir que la estimación del desarrollo humano contiene implícitamente la valoración de esas libertades instrumentales que, como sustrato, alientan o inhiben las libertades denominadas constitutivas o básicas. Y no podría ser de otro modo en tanto que éstas, si bien no se encuentran exclusivamente determinadas por las libertades instrumentales, sí se constituyen y adquieren especificidad en el ambiente o contexto social al que éstas dan forma.

En palabras de Amartya Sen (2000b): "lo que la gente puede lograr positivamente resulta influido por las oportunidades económicas, libertades políticas, poderes sociales, condiciones adecuadas para buena salud y educación básica y el fomento y desarrollo de iniciativas" (p. 16).

En síntesis, implicadas en los indicadores para la medición del Índice de Desarrollo Humano se encuentran condiciones de contexto que, si bien no son parte integrante de él, indudablemente dejan sentir 
su influencia de manera importante. En resumidas cuentas, el IDH, aunque formalmente señala el estado que guardan salud, educación e ingresos en un momento dado, también da cuenta del estado de las libertades y oportunidades existentes en un contexto social integral, y en especial, la situación contextual de las libertades de las niñas y mujeres.

\section{Perspectivas de género}

Las constructoras de la teoría de género advierten sobre la complejidad de algunas de sus categorías entre las que destaca lo que se denomina perspectiva de género (Serret, 2008). Es primordial comprender que, por género, en esta teoría se conceptualiza "el conjunto de creencias, prescripciones y atribuciones que se construyen socialmente tomando a la diferencia sexual como base" (Lamas, s. f.). Esa concepción, de acuerdo con Serret (2008), apoyándose en la distinción biológico-sexual de los cuerpos, terminó por asignar valores y significados inexistentes de manera natural, dando lugar a los "géneros" de las personas.

El enfoque o teoría de género devela a éste como una construcción social, en tanto que lo que las sociedades determinan como "propio" de cada sexo (hombre, mujer) no son atributos que se puedan considerar naturales, "lo cual se pone de manifiesto con claridad, por ejemplo, en el hecho de que cada sociedad define de manera distinta esos mismos conceptos ('hombre' y 'mujer')" (Serret, 2008, p. 51). De acuerdo con Martha Lamas (s. f.), como seres humanos somos iguales (exceptuando la sexualidad y la procreación). Así, la perspectiva de género ha permitido el análisis de las relaciones de poder-subordinación entre mujeres y hombres, y los efectos que ha tenido esta diferenciación en las sociedades. En síntesis:

Cuando se habla de perspectiva de género, se hace alusión a una herramienta conceptual que busca mostrar que las diferencias entre mujeres y hombres se dan no sólo por su determinación biológica, sino también por las diferencias culturales asignadas a los seres humanos... aplicado al proceso de desarrollo visibiliza a las mujeres como sujetos potenciales del desarrollo superando las visiones fragmentadas que las consideran "grupos vulnerables" o ciudadanas de segunda categoría. (INMUJERES, 2007, pp. 104-105)

\subsection{Desigualdad y violencias de género en la Agenda 2030}

La Agenda 2030 para el Desarrollo Sostenible aprobada en 2015 por la ONU, y de la cual México es signatario, contiene 17 objetivos (Objetivos de Desarrollo Sustentable, ODS) y 169 metas que, a manera de hoja de ruta, establecen una perspectiva de acción hacia sociedades económica, social y ambientalmente sustentables y, por definición, de largo plazo e inclusivas, frente a los desafíos que representan los actuales patrones de producción, consumo y, podría agregarse, de convivencia humana, entre otras dimensiones (CEPAL, 2018).

La igualdad de género y el empoderamiento de mujeres y niñas no tan solo es un derecho humano fundamental, se trata, de acuerdo con el Objetivo 5 de la Agenda 2030, de una condición necesaria hacia un mundo pacífico, próspero y sostenible. En la meta 5.2 del Objetivo 5 de la Agenda 2030 se señala: 
"Eliminar todas las formas de violencia contra todas las mujeres y las niñas en los ámbitos público y privado, incluidas la trata y la explotación sexual y otros tipos de explotación”. En tanto, en la meta 16.1 del objetivo 16, Paz y Justicia se menciona: "reducir considerablemente todas las formas de violencia y las tasas de mortalidad conexas en todo el mundo" (CEPAL, 2018, p. 23).

Se reconoce en los ODS, entonces, que la desigualdad y las violencias de género constituyen un freno a los objetivos de desarrollo sostenible y, por la misma razón, contribuyen a la conformación de un entorno adverso al avance del desarrollo humano, expresado en el IDH, y particularmente, del Índice de Desarrollo Humano Relativo al Género.

\subsection{Violencia feminicida y desarrollo humano}

El feminicidio es la muerte violenta de mujeres por razones de género; es "el extremo de la dominación de género contra las mujeres" (Lagarde, 2008, p. 215). El concepto fue acuñado por la antropóloga mexicana Marcela Lagarde (2008) retomando aportes de Diana Russell y Jill Radford, teóricas del feminismo que elaboraron la categoría de femicidio, término éste al que prefirió traducir por feminicidio para evitar la homología con el homicidio; y porque femicidio podría ser interpretado solo como homicidio de mujeres (Lagarde, 2008, p. 216). Traducir de esa manera el concepto permitió identificarlo, en apreciación de la autora, con la sistemática violación a los derechos humanos de las mujeres asesinadas o desaparecidas, así como responsabilizar al Estado por la impunidad en que generalmente terminan. "Hay condiciones para el feminicidio -señala- cuando el Estado (o algunas de sus instituciones) no da las suficientes garantías a las niñas y las mujeres, y no crea condiciones de seguridad que garanticen sus vidas en la comunidad, en la casa, ni en los espacios de trabajo de tránsito o de esparcimiento" (Lagarde, 2008, pp. 216-7). En palabras de Segato (2006):

Es el asesinato de una mujer genérica, de un tipo de mujer, sólo por ser mujer y por pertenecer a este tipo, de la misma forma que el genocidio es una agresión genérica y letal a todos aquellos que pertenecen al mismo grupo étnico, racial, lingüístico, religioso o ideológico. (p. 36)

Los esfuerzos teóricos desarrollados en esta perspectiva han llevado a tipificar, no jurídicamente, el feminicidio. De acuerdo con Iribarne (2015-2016), Diana Russell identificará cuatro tipos sobre la base de la relación existente entre la víctima y el victimario:

- Femicidios de pareja íntima: todos los hombres que tengan o hayan tenido una relación de pareja con la víctima.

- Femicidios de familiares: cualquier pariente masculino ya sea consanguíneamente o político.

- Otros perpetradores conocidos de femicidio: amigos de la familia o de la víctima, colegas masculinos, figuras masculinas de autoridad, conocidos masculinos, citas masculinas (no sexual).

- Femicidio de extraños: extraños masculinos (Russell, 2006, citado en Iribarne, 2015-2016). 
Signatario de diversas convenciones internacionales contra la violencia de mujeres y niñas, el Estado mexicano ha generado un marco jurídico que reconoce esa violencia y garantiza una vida libre de ella. Tales son:

- Ley General de Acceso de las Mujeres a una Vida Libre de Violencia (LGAMVLV) (Congreso de la Unión, 2007, 1 de febrero). Garantiza y protege el derecho de las mujeres a una vida libre de violencia.

- Reforma en materia de derechos humanos al artículo primero de la Constitución Política de los Estados Unidos Mexicanos (CPEUM) (Congreso de la Unión, 1917, 5 de febrero). Incorpora y eleva a rango constitucional los derechos humanos derivados de los tratados internacionales ratificados por México.

- El Código Penal Federal (CPF) (Congreso de la Unión, 1931, 14 de agosto) tipifica al feminicidio como delito autónomo.

Se tipifica el feminicidio en el Código Federal de la siguiente manera:

Artículo 325. Comete el delito de feminicidio quien prive de la vida a una mujer por razones de género. Se considera que existen razones de género cuando concurra alguna de las siguientes circunstancias:

I. La víctima presente signos de violencia sexual de cualquier tipo;

II. A la víctima se le hayan infligido lesiones o mutilaciones infamantes o degradantes, previas o posteriores a la privación de la vida o actos de necrofilia;

III. Existan antecedentes o datos de cualquier tipo de violencia en el ámbito familiar, laboral o escolar, del sujeto activo en contra de la víctima;

IV. Haya existido entre el activo y la víctima una relación sentimental, afectiva o de confianza; V. Existan datos que establezcan que hubo amenazas relacionadas con el hecho delictuoso, acoso o lesiones del sujeto activo en contra de la víctima;

VI. La víctima haya sido incomunicada, cualquiera que sea el tiempo previo a la privación de la vida;

VII. El cuerpo de la víctima sea expuesto o exhibido en un lugar público (CPF, 2019)

El feminicidio constituye un fenómeno global que se ha incrementado de manera alarmante, incluido nuestro país. En este contexto, los ojos del mundo fueron puestos en México a inicios de la década de los 90, con los asesinatos de mujeres en Ciudad Juárez.

En la actualidad son distintas las fuentes de información en que se apoyan investigaciones sobre el tema. Registros administrativos sobre defunciones (INEGI, 2018), estadísticas basadas en información de las Procuradurías Estatales concentradas por el Secretariado Ejecutivo del Sistema de Seguridad Nacional (SESNSP), o seguimientos periodísticos por parte de organizaciones de la sociedad civil, son algunas de ellas.

Información gestionada por el Secretariado Ejecutivo del Sistema de Seguridad Nacional, indican que los feminicidios muestran una tendencia ascendente consistente entre los años de 2015 a 2019, pasando 
de 411 en el primer año a 604 en 2016, 741 en 2017, 891 en 2018 y 983 en 2019 (Sistema Nacional de Seguridad Pública, 2020).

Estudios que se apoyan en las estadísticas vitales del INEGI asumen como variable proxy a los feminicidios de las Defunciones Femeninas con Presunción de Homicidio (DFPH), de tal manera que los registros estadísticos ofrecen la posibilidad de analizar una tendencia históricamente ascendente desde 1985 (Echarri, 2017).

Las desigualdades de género han sido abordadas para establecer las brechas existentes en los propios indicadores del IDH (PNUD, 2014); con relación a la pobreza (CONEVAL, 2012); a los derechos humanos y las políticas públicas (Facio, 2003); a la salud (Secretaría de Salud, 2005), entre otros distintos enfoques.

Estudios sobre la violencia de género y su factor de impacto negativo sobre el IDH han llegado a la conclusión de que la violencia contra la mujer limita "sus libertades para elegir, para hacer uso de sus capacidades, para potenciarlas y tener proyectos propios" (De la Paz, 2007, p. 14), que la violencia ejercida contra ellas implica una pérdida de autonomía para ejercer sus derechos, lo cual implica que, "cuando la autonomía de las personas se reduce, el valor que dan al conjunto de oportunidades para ser o hacer también se reduce" (PNUD, 2016, p. 3). Tales investigaciones han contribuido a la valoración de la violencia contra las mujeres en el IDH. Específicamente han generado un IDH ajustado por violencia contra las mujeres, en el ámbito doméstico o de pareja (PNUD, 2016).

\section{Conclusiones}

Las construcciones teóricas analizadas se abrieron paso en décadas recientes para colocarse como referentes ineludibles en cada uno de sus campos. El desarrollo sustentable es una propuesta que al incorporar las dimensiones económicas, sociales y ambientales dejó atrás concepciones basadas preferentemente en el crecimiento económico; el enfoque del desarrollo humano como marco para la estimación del avance de las sociedades en términos de bienestar social, termina por desplazar la preeminencia que ocupaba el ingreso per cápita en esta tarea; finalmente, la teoría de género, y su riqueza conceptual, al cuestionar fuertemente la dominación patriarcal y la violencia de género, pasa a ocupar, no sin obstáculos, un lugar legitimado en instituciones gubernamentales, academias y entre la sociedad.

De esta manera, las contribuciones de la teoría de género, sacaron a la luz el hecho de que los roles y patrones de comportamiento basadas en el sexo de las personas son construcciones sociales, y no precisamente consecuencias naturales; que tales construcciones atribuyeron a la mujer un papel subordinado en una sociedad dominada por concepciones masculinas, sobre las que se dieron profundas desigualdades en acceso a oportunidades y ejercicio de derechos entre ambos sexos. La teoría de género se encargó de visibilizar esta situación, siendo una de sus mayores contribuciones. De aquí que, pocos años después de iniciada la estimación del IDH en 1990, se incorporen mediciones para develar tales desigualdades por medio del IDH relativo al Género (IDHrG), y colocarlas como focos de atención para las instituciones de gobierno respectivas.

Así, en el marco de la teoría de género emergieron líneas de análisis que profundizaron y especificaron las desigualdades en distintos órdenes de la vida social, con desventajas para las mujeres. En este sentido, 
pueden señalarse las contenidas en el IDHrG (salud, ingreso y educación), pero también pueden apuntarse estudios sobre pobreza, sexualidad, participación política, uso del tiempo, trabajo doméstico, etc.

Entonces, el feminicidio es situado como un hecho que obstaculiza y limita la agencia de las mujeres, su libertad y capacidad de actuar, en la medida en que la ocurrencia generalizada del mismo genera o fortalece una condición de vulnerabilidad ante el peligro de sufrir tal violencia. Que ésta crea un ambiente social que obstruye proyectos de vida, limitando los horizontes por los que podrían transitar las niñas y mujeres y, por lo mismo, restringe el desarrollo de capacidades.

El feminicidio deteriora las libertades instrumentales ya referidas, impactando, indirectamente si se quiere, la consecución de logros o avances que el IDHrG recoge para su evaluación. Pudiéndose rescatar entonces que, en el nivel teórico, existe una relación entre el feminicidio y el IDHrG.

Ahora bien, conceptualizada la sustentabilidad social como el proceso mediante el cual la población crecientemente fortalece su bienestar, estando éste vinculado al desarrollo de capacidades en un marco de libertad para ejercer derechos inalienables, resulta pertinente establecer que, en tanto niñas y mujeres se encuentren sometidas a las violencias que sobre ellas se ejercen, incluida su forma extrema, aquel proceso que direcciona hacia la sustentabilidad social encontrará formidables obstáculos. Es aquí donde se encuentra soporte al planteamiento de que los tres constructos teóricos que se presentan tienen puntos que las articulan.

Finalmente, los objetivos 5 de Igualdad de Género y 11 de Paz y justicia de la Agenda 2030, orientan normativamente la investigación social para explicar el factor de impacto negativo de la violencia de género sobre el desarrollo humano de niñas y mujeres, avanzando así en una visión más realista sobre el desarrollo sustentable con perspectiva de género, para formular políticas públicas que incidan en la solución de tan grave problema sistémico de nuestras sociedades, y en particular de México.

\section{Referencias}

Alkire, S. (2005). Subjective Quantitative Studies of Human Agency. Social Indicators Research, 74, 217-260. Alkire, S. (2008). Concepts and measures of agency. OPHI Working Paper. https://ophi.org.uk/workingpaper-number-09/

Comisión Económica para América Latina y el Caribe. (2018). Agenda 2030 y los objetivos de desarrollo sostenible. Una oportunidad para América Latina y el Caribe. Objetivos, metas e indicadores mundiales. CEPAL. https://www.cepal.org/es/publicaciones/40155-la-agenda-2030-objetivos-desarrollosostenible-oportunidad-america-latina-caribe

Congreso de la Unión. (1917, 5 de febrero). Constitución Política de los Estados Unidos Mexicanos. DOF 15 de mayo de 2019. http://www.diputados.gob.mx/LeyesBiblio/index.htm

Congreso de la Unión. (1931, 14 de agosto). Código Penal Federal. DOF 12-04-2019. http://www.diputados.gob.mx/LeyesBiblio/index.htm

Congreso de la Unión. (2007, 1 de febrero). Ley general de acceso de las mujeres a una vida libre de violencia. DOF 13-04-201. http://www.diputados.gob.mx/LeyesBiblio/index.htm

Consejo Nacional de Evaluación de la Política de Desarrollo Social. (2012). Pobreza y género en México. Hacia un sistema de indicadores. Información 2008-2012. CONEVAL. https://www.coneval.org.mx/ Medicion/MP/Paginas/Pobreza-y-genero-en-Mexico-2010-2016.aspx 
De la Cruz, C. (2007). Género, derechos y desarrollo humano. Programa de las Naciones Unidas para el Desarrollo San Salvador. http://www.dhls.hegoa.ehu.eus/documents/4872

De la Paz, B. (2007). Desarrollo humano y violencia contra las mujeres en Zacatecas. Programa de las Naciones Unidas para el Desarrollo. http://cedoc.inmujeres.gob.mx/insp/desarrollo.pdf

Echarri, C. J. (2017). La violencia feminicida en México, aproximaciones y tendencias 1985-2016. Características, tendencias y nuevas expresiones en las entidades federativas. ONU-Mujeres, INMUJERES, CÁMARA DE DIPUTADOS. https://www.gob.mx/cms/uploads/attachment/file/293666/ violenciaFeminicidaMx_07dic_web.pdf

Facio, A. (2003). Los derechos humanos desde una perspectiva de género y las políticas públicas. Otras Miradas, 3(1), 15-26.

Foladori, G. y Tommasino, H. (2000). El concepto de desarrollo sustentable treinta años después. Desenvolvimento e Meio Ambiente, (1), 41-56.

Fougeyrollas, P. (1980). Los procesos sociales contemporáneos. Fondo de Cultura Económica.

Folbre, N. (2006). Measuring Care: Gender, Empowerment, and the Care Economy. Journal of Human Development, 7(2), 183-199.

Frank, A. G. (1967). El desarrollo del subdesarrollo. Pensamiento Crítico, (7), 159-173.

Hernández Cortez, N., Ruelas Monjardín, L. C. y Nava Tablada, M. E. (2018). Sustentabilidad del desarrollo: desafíos y propuestas. Secretaría de Educación de Veracruz.

Instituto Nacional de Estadística y Geografía. (2018). Estadísticas Vitales. Defunciones Registradas 2018. INEGI. https://www.inegi.org.mx/rnm/index.php/catalog/529

Instituto Nacional de la Mujeres. (2007). Glosario de género. INMUJERES. http://cedoc.inmujeres.gob. $\mathrm{mx} /$ documentos_download/100904.pdf

Iribarne, M. (2015-2016). Feminicidio (en México). Eunomía. Revista en Cultura de la Legalidad, (9), 205-223.

Kovel, J. y Löwy, M. (2001). Manifiesto ecosocialista. Systemic Alternatives. https://systemicalternatives. org/2014/03/05/manifiesto-ecosocialista/

Lagarde, M. (2008). Antropología, feminismo y política: violencia feminicida y derechos humanos de las mujeres. En M. Bullen y C. Diez (Coords.), Retos teóricos y nuevas prácticas (pp. 209-239). Ankulegi.

Lamas, M. (s. f.). El género es cultura. http://www.paginaspersonales.unam.mx /app/webroot/files/981/ El_genero_es_cultura_Martha_Lamas.pdf

López-Calva, L. y Vélez, R. (2003). El concepto de desarrollo humano, su importancia y aplicación en México. Estudios Sobre Desarrollo Humano PNUD México No. 2003-1. https://sic.cultura.gob.mx/ documentos/1007.pdf

Nussbaum, M. C. y Sen, A. (Comps.) (2004). La calidad de vida. Fondo de Cultura Económica.

Organización de las Naciones Unidas. (1987). Informe Brundtland. ONU. https://undocs.org/es/A/42/427

Picazzo, E., Gutiérrez, E., Infante, J. y Cantú, P.C. (2011). La teoría del desarrollo humano y sustentable: hacia el reforzamiento de la salud como un derecho y libertad universal. Estudios sociales, 19(37), 253-279.

Programa de las Naciones Unidas para el Desarrollo. (1995). Informe sobre Desarrollo Humano 1995. PNUD. http://hdr.undp.org/sites/default/files/hdr_1995_es_completo_nostats.pdf

Programa de las Naciones Unidas para el Desarrollo. (2003). Informe sobre Desarrollo Humano México 
2002. PNUD MÉXICO. http://hdr.undp.org/sites/default/files/mexico_2002_sp.pdf

Programa de las Naciones Unidas para el Desarrollo. (2009). Indicadores de desarrollo humano y género en México. 2000-2005. INMUJER ESPAÑA. http://www.inmujer.gob.es/publicacioneselectronicas/ documentacion/Documentos/DE1077.pdf

Programa de las Naciones Unidas para el Desarrollo. (2014). Indicadores de desarrollo humano y género en México. Identificar las barreras para lograr la igualdad. PNUD MÉXICO. https://www.undp.org/ content/dam/mexico/docs/Publicaciones /PublicacionesReduccionPobreza/InformesDesarrolloHumano/DHyG\%20baja\%20res.pdf

Programa de las Naciones Unidas para el Desarrollo. (2015). ¿Qué es el desarrollo humano? PNUD. http:// hdr.undp.org/en/node/2228

Programa de las Naciones Unidas para el Desarrollo. (2016). Desarrollo humano y violencia contra las mujeres en México. PNUD América Latina y El Caribe. https://www.latinamerica.undp.org/content/rblac/es/home/library/womens_empowerment/desarrollo-humano-y-violencia-contra-lasmujeres-en-mexico--.html

Rostow, W. (1965). Las etapas del crecimiento económico. Un manifiesto no comunista. Fondo de Cultura Económica.

Ruppanner, L. E. (2010). Cross-national reports of housework: An investigation of the gender empowerment measure. Social Science Research, 39, 963-975.

Secretaría de Salud (2005). Género y Salud. Una introducción para tomadores de decisiones. Centro Nacional de Equidad de Género y Salud Reproductiva. http://www.cdi.salud.gob.mx:8080/BasesCDI/ Archivos/Equidaddegenero/DOCSAL7598.pdf

Segato. R. L. (2006). La escritura en el cuerpo de las mujeres asesinadas en Ciudad Juárez. Territorio, soberanía y crímenes de segundo estado. Tinta Limón.

Sen, A. (1999). Development as Freedom. Blackwell.

Sen, A. (2000a). Desarrollo y libertad. Planeta.

Sen, A. (2000b). El desarrollo como libertad. Gaceta Ecológica, (55), 14-20.

Sen, A. (2009). The Idea of Justice. Harvard University Press.

Sen, A. (2010). La idea de la justicia. Taurus.

Serret Bravo, E. (2008). Qué es y para qué es la perspectiva de género. Libro de texto para la asignatura: Perspectiva de Género, en educación superior. Instituto de la Mujer Oaxaqueña del Gobierno Constitucional del Estado de Oaxaca. Colección Instituto de la Mujer Oaxaqueña Ediciones, serie Buenas Prácticas.

Sistema Nacional de Seguridad Pública (2020). Información sobre violencia contra las mujeres. Gobierno de México. https://drive.google.com /file/d/1eJvQGWG3Fe9Xls53DpfCArCt5ARd-FbN/view

Vergara Tamayo, C. A. y Ortiz Mota, D. C. (2016). Desarrollo sostenible: enfoques desde las ciencias económicas. Revista Apuntes del CENES, 35(62), 15-52. 